\title{
Precision Material Modification and Patterning with Helium Ions
}

\author{
David C. Bell*, Max Lemme** and Charles M. Marcus** \\ * School of Engineering and Applied Sciences and the Center for Nanoscale \\ Systems, Harvard University, Cambridge MA 02138 \\ ** Department of Physics, Harvard University, Cambridge MA 02138
}

The Helium ion microscope has been primarily developed as an imaging tool $[1,2]$, but being a charged ion beam instrument it is also possible to perform milling and sputtering tasks more commonly associated with a conventional Ga ion beam system (FIB). One distinct advantage of a Helium ion system is milling and sputtering of soft materials with the extremely low milling and sputtering rates. The Helium ion microscope also boasts an extremely small probe size in the order of $0.5 \mathrm{~nm}$ or better. The combination of the abovementioned features makes this system one of the most precise direct fabrication tools currently available for suitable materials, for example graphene. Graphene is a two dimensional carbon crystal that is of particular interest due to its potential applications in electronic and optical devices. Suspended graphene has also shown high electron mobility and strength, properties important in prospective microelectronics devices. Use of HeIM for nanosculpting and patterning of graphene will present an avenue for better device fabrication with novel physical properties.

There has been much interest in the formation of nanopores for development of methods of DNA and protein sequencing. It is possible to fabricate nanopores using the He ion beam. This is attractive since the pores produced do not suffer from ionic contamination. Nanopores can also be fabricated in substrates that have thin metal layers (even multiple layers); such layers can be either thermally or e-beam evaporated onto a membrane. One application of interest is to fabricate a nanoscale fuel cell, using ionic flow through nanopores. An example of a Pt membrane with nanopores is shown below in Figure 1. Since the pore size is controlled and can be made uniformly symmetrical, such pores can also be used as apertures in near-field optical instruments and semiconductor devices.

In addition to employing resists like in the conventional lithography manner [3], there are advantages in patterning directly due to the interaction volume of the $\mathrm{He}$ ion beam and the physical interaction mechanisms (Figure 2). We have employed a Nabilty pattern generation system (NPGS) in the Orion HeIM to produce milling, etching test patterns and devices. We will present results of sputtering and milling rates of different materials and examples of precision milling and ion etching.

\section{References.}

[1] J. Morgan, J. Notte, R. Hill, B. Ward "An Introduction to the Helium Ion Microscope", Microscopy Today (2006), vol. 14 (4), pp. 24-31. 
[2] D.C. Bell "Contrast Mechanisms and Image Formation in Helium Ion Microscopy", Microscopy and Microanalysis in press (2009).

[3] D. Winston et al "Helium-ion Lithography with Hydrogen Silsesquioxane Resist", Proc. EIBPN 2009.

[4] We would like to thank Louis Stern, Dave Vocci and Ed Marchbanks of the Carl Zeiss SMT, ALIS Business Unit for his help and support with this project.
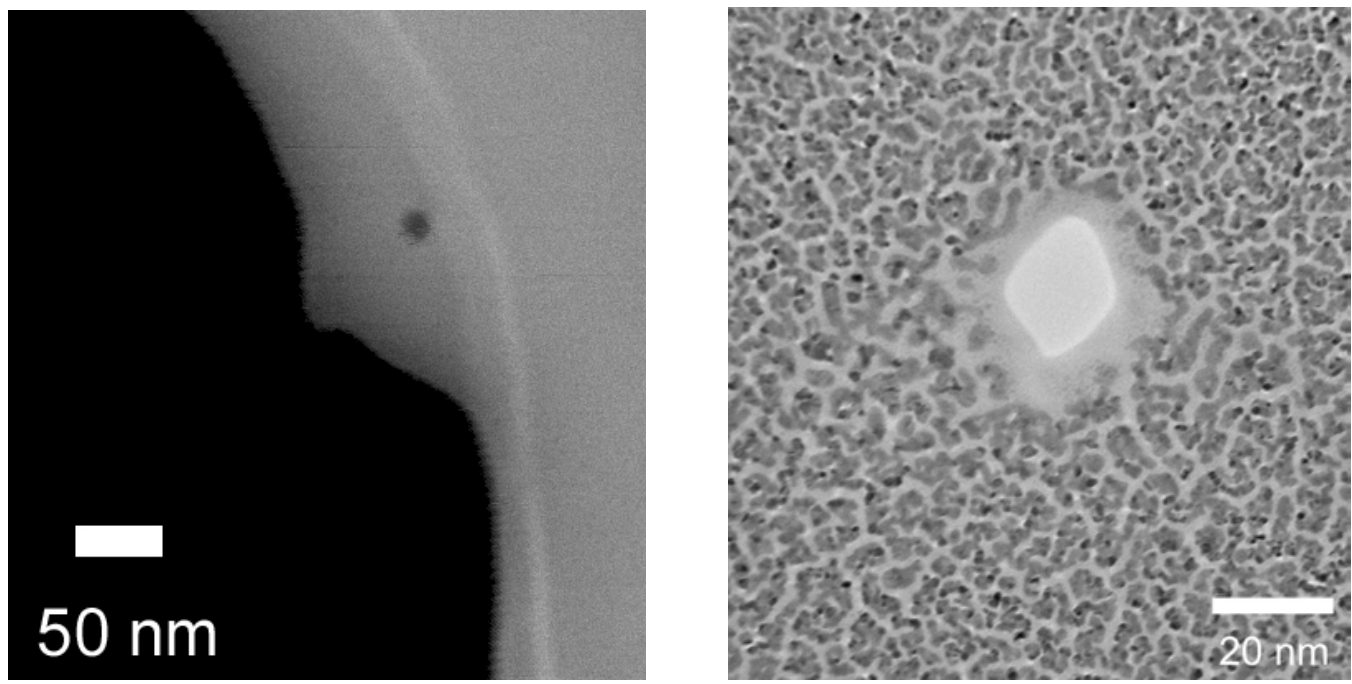

Figure 1. Left, nanopore drilled in a carbon based substrate. Right, nanopore drilled with the Helium ion beam in a metalized layer coated SiN membrane.

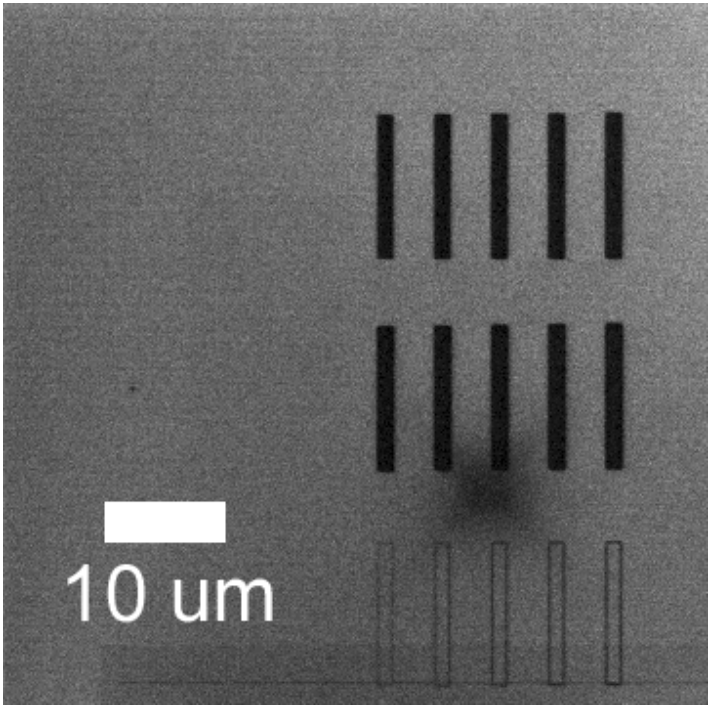

Figure 2. Direct writing etched patterns in $\mathrm{SiO}$ layer, indicating the ability to remove material and etch thin lines in suitable substrates. 